\title{
Late Metastatic Renal Cell Carcinoma Recurrence Presenting as a Maxillary Sinus Mass 12 Years after Nephrectomy: Case Report and Literature Review
}

\author{
Yeon-Hee Joo, $\mathrm{MD}^{1,3}$, Yung Jin Jeon, $\mathrm{MD}^{2}$, Hyun-Jin Cho, $\mathrm{MD}, \mathrm{PhD}^{2}$, and Sang-Wook Kim, $\mathrm{MD}, \mathrm{PhD}^{2,3}$ \\ ${ }^{I}$ Department of Otorhinolaryngology, Gyeongsang National University Changwon Hospital and \\ Gyeongsang National University College of Medicine, Changwon; and \\ ${ }^{2}$ Department of Otorhinolaryngology, Gyeongsang National University Hospital and \\ Gyeongsang National University College of Medicine, Jinju; and \\ ${ }^{3}$ Institute of Health Sciences, Gyeongsang National University, Jinju, Korea
}

\begin{abstract}
Metastasis to the sinonasal cavity is rare. Late recurrence, such as metastasis developing 10 years or more after nephrectomy, is even more rare. We present a rare case of late metastatic renal cell carcinoma (RCC) in the maxillary sinus after nephrectomy and discuss reported sinonasal metastatic RCC cases. A 64-year-old man presented with left nasal obstruction for the previous one year. He had undergone right nephrectomy to treat RCC 12 years prior. Paranasal sinus computed tomography and magnetic resolution imaging revealed a 4.6-cm-diameter mass exhibiting delayed contrast enhancement that filled the left maxillary sinus and the nasal cavity. Histologically, clear cell RCC was diagnosed. We resected the maxillary sinus tumor to resolve the nasal obstruction for palliative symptom relief. There has been no evidence of recurrence to date, 3 years postoperatively. When a patient with a history of RCC presents with a hypervascular paranasal sinus mass, metastatic RCC should be considered, despite history of nephrectomy. It is important to make an appropriate treatment plan depending on extent of metastases and location of the metastatic tumor.
\end{abstract}

KEY WORDS: Renal cell carcinoma $\cdot$ Metastasis $\cdot$ Paranasal sinus $\cdot$ Nephrectomy.

\section{INTRODUCTION}

Renal cell carcinoma (RCC) is aggressive and represents $3 \%$ of all malignant tumors; $25-30 \%$ show overt metastases at the initial presentation, often to the lungs, bone, and liver. ${ }^{12)}$ The 5-year survival rate for patients with RCC confined to the kidney is about $95 \%$, whereas that for patients with metastatic RCC it is only $0-20 \%{ }^{3 / 4)}$ The nasal cavity and paranasal sinus are rare metastatic sites; very few cases have been reported, although RCC is the malignancy that most commonly metastasizes to the nasal cavity and paranasal sinus. ${ }^{56)}$ Delayed metastasis can also occur. Such metastases are extremely rare, and may onset several years after resection of the primary renal lesion without primary tumor recurrence. There are various options for treating metastatic RCC, such as surgical excision, radiotherapy, chemotherapy, and immunotherapy. The type of metastasis (solitary or multiple) and location of the metastatic tumor are important when making treatment decisions. However, no treatment guidelines for metastatic sinonasal RCC have been established, mainly because metastatic sinonasal RCC is rare. Thus, rhinologists are dependent on case reports. We report a rare case of metastatic RCC presenting as a maxillary sinus mass after nephrectomy 12 years prior. We summarize the literature on metastatic RCC to the sinonasal cavity more than 10 years after nephrectomy, with the goal of establishing a treatment strategy.

Received: October 26, 2020 / Revised: December 24, 2020 / Accepted: December 24, 2020

Address for correspondence: Yeon-Hee Joo, MD, Department of Otorhinolaryngology, Gyeongsang National University Changwon Hospital, 11 Samjeongja-ro, Seongsan-gu, Changwon 51472, Korea

Tel: +82-55-214-3863, Fax: +82-55-214-3262, E-mail: yhjoo@gnuh.co.kr 


\section{CASE REPORT}

A 64-year-old man presented with a left nasal obstruction 1 year earlier. Twelve years earlier, an $8 \mathrm{~mm}$ tumor was found in his right kidney and he was diagnosed with RCC stage I. He had undergone right nephrectomy to treat the $\mathrm{RCC}$ and no recurrence had been noted during the 10-year follow-up. On endoscopic examination, both nasal cavities were narrowed; left inferior turbinate hypertrophy was observed and the nasal septum was deviated to the right (Fig. 1). Paranasal sinus computed tomography (CT) revealed a round 4.6-cm-diameter tumor occupying the entire paranasal sinus and nasal cavity; this tumor caused deviation of the septum to the right (Fig. 2A). Paranasal sinus magnetic resonance imaging (MRI) revealed an enhanced hypervascular round mass and sinusitis (Fig. 2B). We planned an endoscopic biopsy and simultaneous complete resection of the nasal cavity mass if possible. During the operation, the bleeding was too severe to allow us to remove the entire sinus mass, and we stopped the operation after partial resection. Histologically, metastatic clear cell RCC in the maxillary sinus tumor was diagnosed. Characteristic clear cytoplasmic cells arranged in nests with intervening blood vessels were observed by H\&E staining. Immunohistochemistry showed increased expression of CD10 and vimentin (Fig. 3). Positron emission tomography CT revealed multiple metastases, including masses in the left femur, abdomen, left arm, and lumbar spine. Rhinology surgeons, an oncologist, and a radiation oncologist discussed a treatment plan. Although this patient had systemic metastasis, local control through surgery was necessary because the chief complaint was nasal obstruction and the potential bleeding risk was high due to the hypervascular nature of the tumor. At the previous surgery, the surgeon noted that the tumor was well encapsulated and dissected, although the surgery was stopped due to severe bleeding. Considering these operative findings, localized tumor, and the need for palliative therapy, endoscopic resection was planned after embolization. The patient underwent angiography and embolization. A feeding artery from the left internal maxillary artery and ethmoidal branch of the left ophthalmic artery were identified, and the vessels inside the maxillary sinus tumor were well enhanced with contrast during angiography. The feeding vessels from the distal internal maxillary artery were embolized with polyvinyl alcohol particle. The thick feeding artery was embolized with a fiber coil to reduce flow. The contrast enhancement from the ethmoidal branch was slight, and embolization was not performed considering the risk of complications. Thereafter, we re-operated to remove the remaining tumor for palliative treatment, to relieve the nasal obstruction and reduce the potential bleeding risk.

The nasal cavity tumor was totally resected via endoscopic medial maxillectomy. The lateral nasal wall was already eroded, and had thinned due to the pressure exerted by the huge mass. After piecemeal resection of the anterior portion of the tumor using cutting and grasping forceps, the overall tumor extent and relationship with the surrounding structures were identified. The amount of bleeding was tolerable compared to the previous surgery due to the effects of embolization. The tumor was well-encapsulated, so the

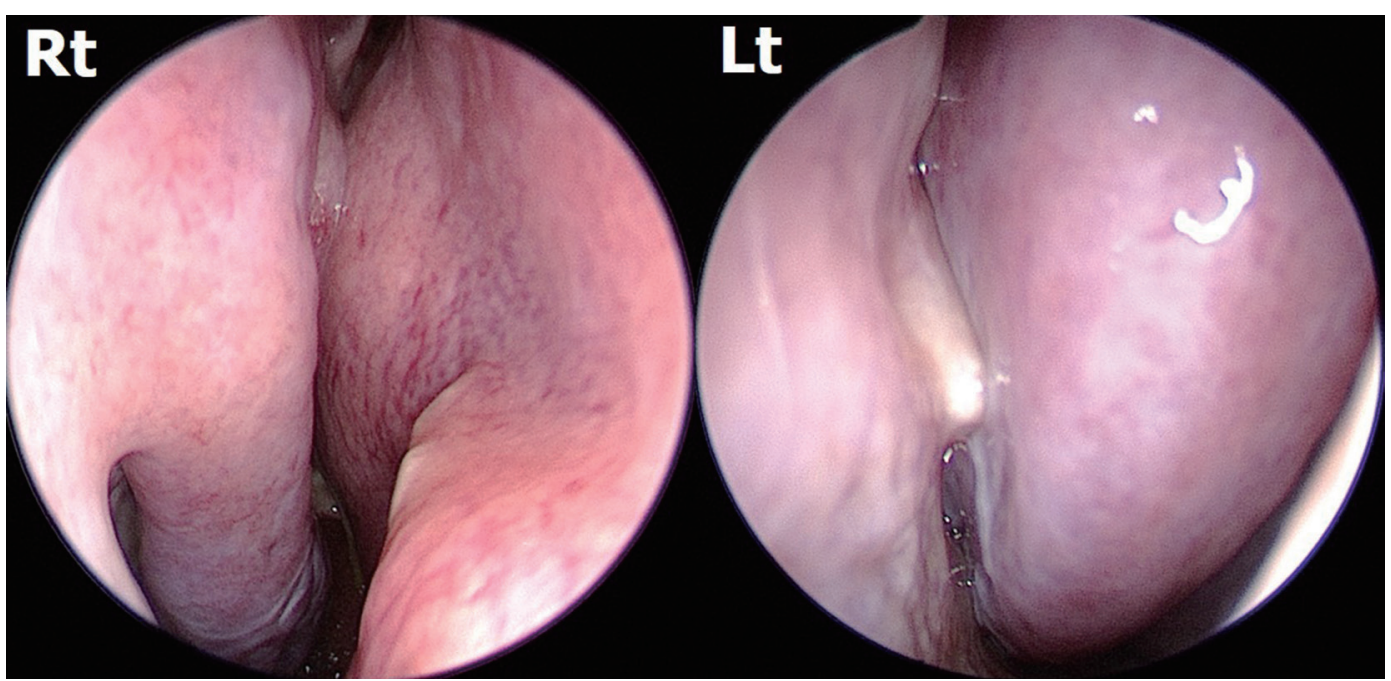

Fig. 1. The nasal septum is deviated to the right and left inferior turbinate hypertrophy is evident. 
remnant tumor was easily dissected from the septum, and anterior and lateral walls of the maxillary sinus, using a Freer elevator and curved instruments without a drill. In the posterior wall of the maxillary sinus, a feeding artery from a branch of the internal maxillary artery was identified, cau- terized, and then cut. After complete resection of the tumor, the tumor margins were clear, and a frozen section of the tumor margin showed no tumor. The posterior part of the nasal septum was perforated because of bony erosion caused by the extended mass. However, the adjacent mucosa was
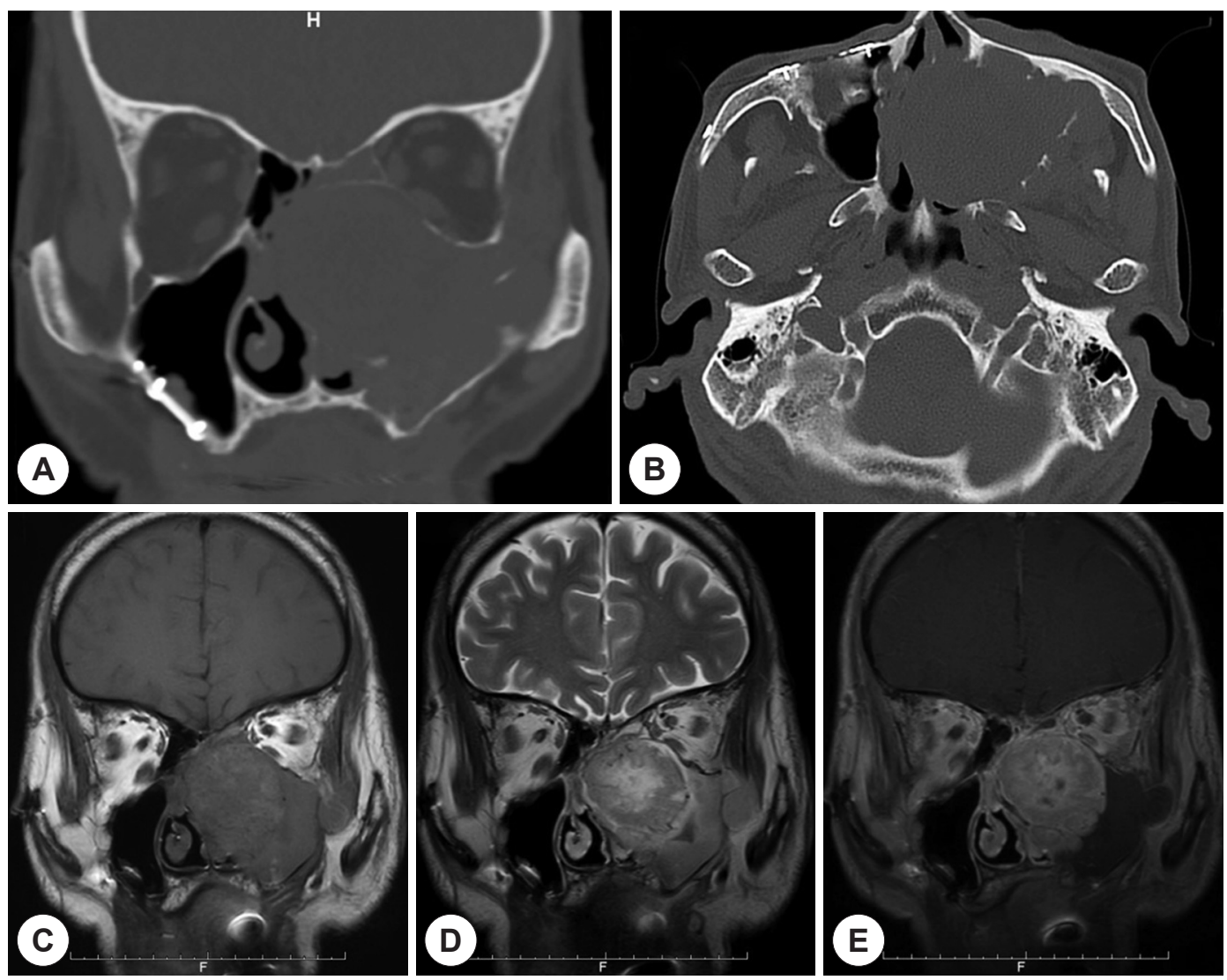

Fig. 2. Preoperative paranasal sinus CT and MRI. A: Coronal CT view reveals a huge mass in the left maxillary sinus and displacement of the bony nasal septum to the right, accompanied by erosion. B: The axial CT view shows the thinned left maxillary sinus posterior wall. C: T1 MRI shows a huge mass occupying the left maxillary and ethmoid sinuses. D: The T2 image shows a well-circumscribed round mass and secondary maxillary sinusitis. E: Enhanced TI MRI reveals a well-enhanced round hypervascular mass and secondary left maxillary sinusitis.
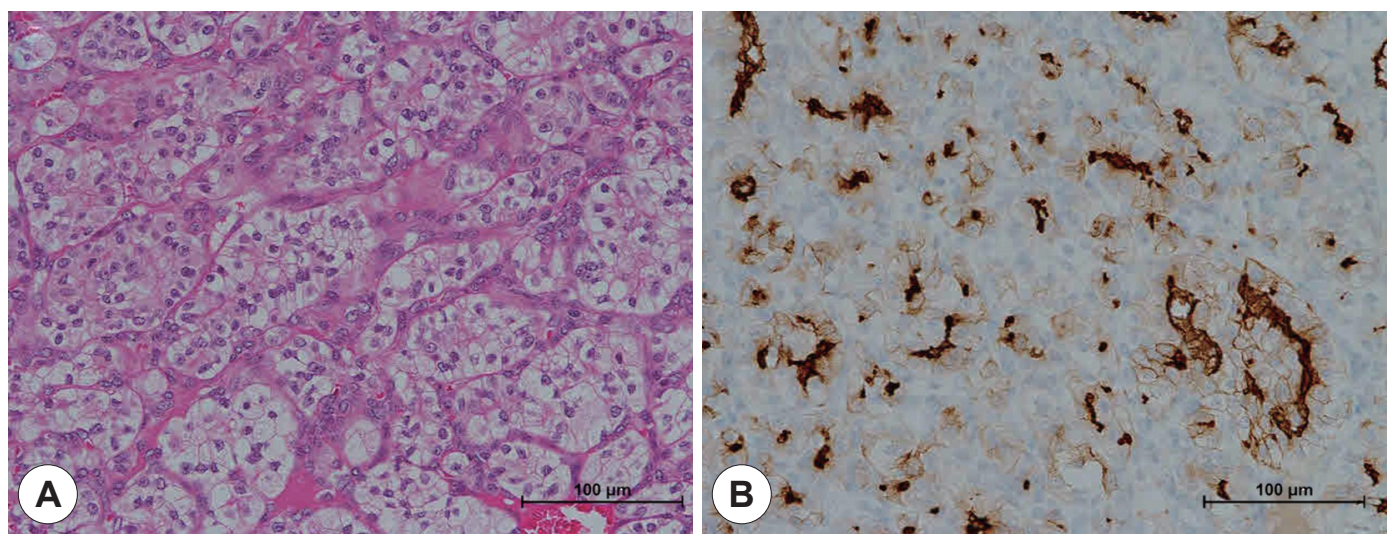

Fig. 3. Histological findings. Histological examination reveals RCC cells with characteristic clear cytoplasm and neoplastic cells arranged in nests with intervening blood vessels on H\&E staining $(A, \times 200)$. Immunohistochemistry for CDI0 showed increased expression $(B, \times 200)$. 

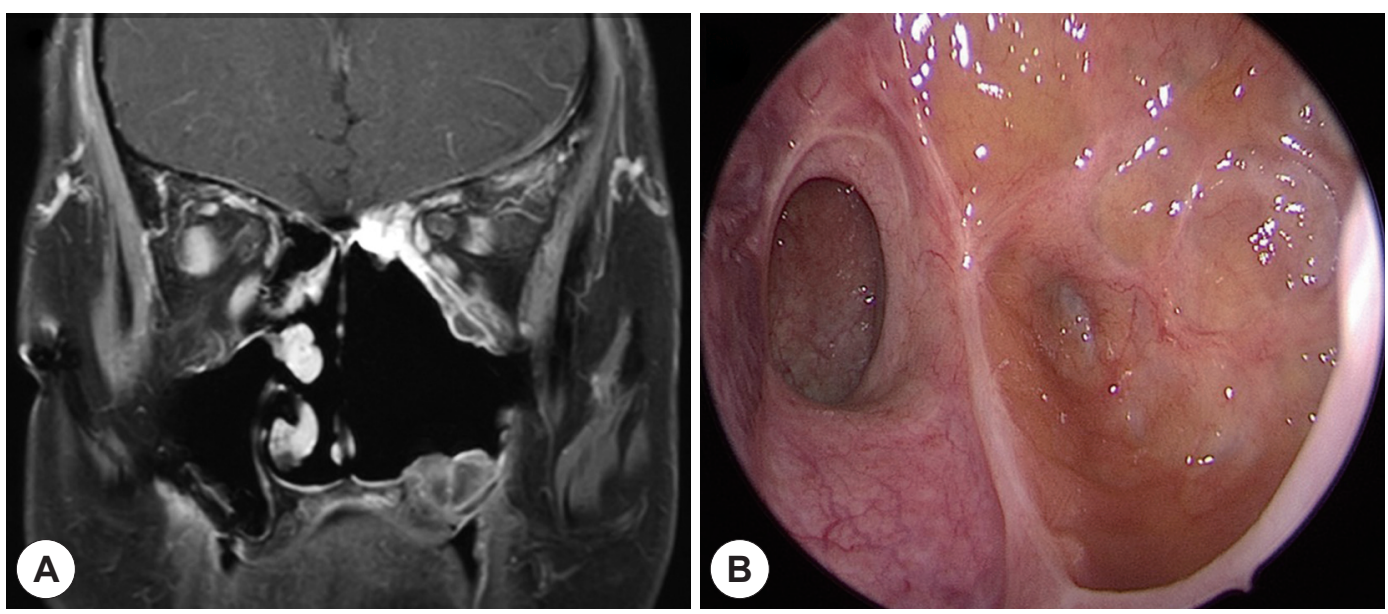

Fig. 4. Postoperative paranasal sinus $C T$ and endoscopic view. A: Postoperative paranasal sinus MRI revealed no residual mass and a clear sinus. B: After medial maxillectomy, endoscopy revealed a clear maxillary sinus and a well-healed sinus mucosa. No residual mass was evident.

clear without erosion or invasion. The histological data were consistent with metastatic clear cell type RCC. We prescribed pazopanib immunotherapy. However, the metastasis in the left femur has progressed and internal fixation was performed 3 years after diagnosing systemic metastasis. Axitinib was prescribed as second-line chemotherapy instead of pazopanib. There is no evidence of recurrence of the maxillary sinus RCC to date, 3 years after the surgery (Fig. 4).

\section{DISCUSSION}

The incidence of RCC is rising and most RCCs are clear cell carcinomas. ${ }^{7)}$ About $20 \%$ of RCC patients develop metastatic disease after diagnosis of early-stage disease. ${ }^{8)}$ Furthermore, in $7.5 \%$ of patients, a metastatic tumor may be the initial presentation leading to diagnosis of the primary tumor." Although metastasis is relatively common, nasal cavity and paranasal sinus metastases are rare, comprising fewer than $1 \%$ of all RCC metastases.

RCC metastasize to the sinonasal cavity via two potential routes: the first is through the inferior vena cava, lungs, heart, and maxillary artery, and the second is through the venous anastomoses of the vertebral venous plexus, intracranial venous plexus, and cavernous venous plexus. Ultimately, tumor cells can reach the sinonasal cavity via these two routes. ${ }^{9)}$ Only a few cases of late metastasis to the sinonasal region have been reported. Late metastasis in the absence of primary tumor recurrence is rarer. Table 1 shows reported cases of metastatic sinonasal RCC without primary tumor recurrence (Table 1). ${ }^{10-13)}$
Interestingly, about $20-30 \%$ of patients develop metastases after curative nephrectomy, usually within 5 years and about $11 \%$ of patients exhibit late recurrence, 10 years or more after nephrectomy. ${ }^{1415)}$ Friberg proposed mechanisms for late metastasis in cancer. ${ }^{16)}$ After the primary tumor has been removed, metastatic cells can remain dormant for decades and dormancy breaking can lead to late recurrence. In some RCCs, the growth rate is very slow and it might take decades for a single cell to multiply so that the tumor is large enough to diagnose.

In patients with sinonasal metastases, the nasal cavity is most commonly affected. ${ }^{17)}$ Epistaxis is the most common symptom, followed by nasal obstruction. Our present patient also complained of nasal obstruction. However, he did not present with epistaxis because the tumor was well-encapsulated despite exhibiting hypervascularity on both MRI and histologically. Metastatic RCC in the sinonasal cavity presents as highly contrast-enhanced round masses on CT and MRI. The differential diagnoses of a hypervascular mass in the nasal cavity or paranasal sinuses include angiofibroma, hemangiopericytoma, hemangioma, and sinonasal glomus tumors. Malignant lesions should also be considered, such as melanomas, adenocarcinomas, and other metastatic tumor. ${ }^{18)}$ Because a hypervascular mass has a risk of massive bleeding, any biopsy must be performed carefully and general anesthesia may be required. Preoperative embolization can help to reduce bleeding during tumor resection.

Treatment of metastatic RCC is palliative in the majority of patients and varies by tumor location, number of metastasis, and primary tumor status. Unlike other metastatic 


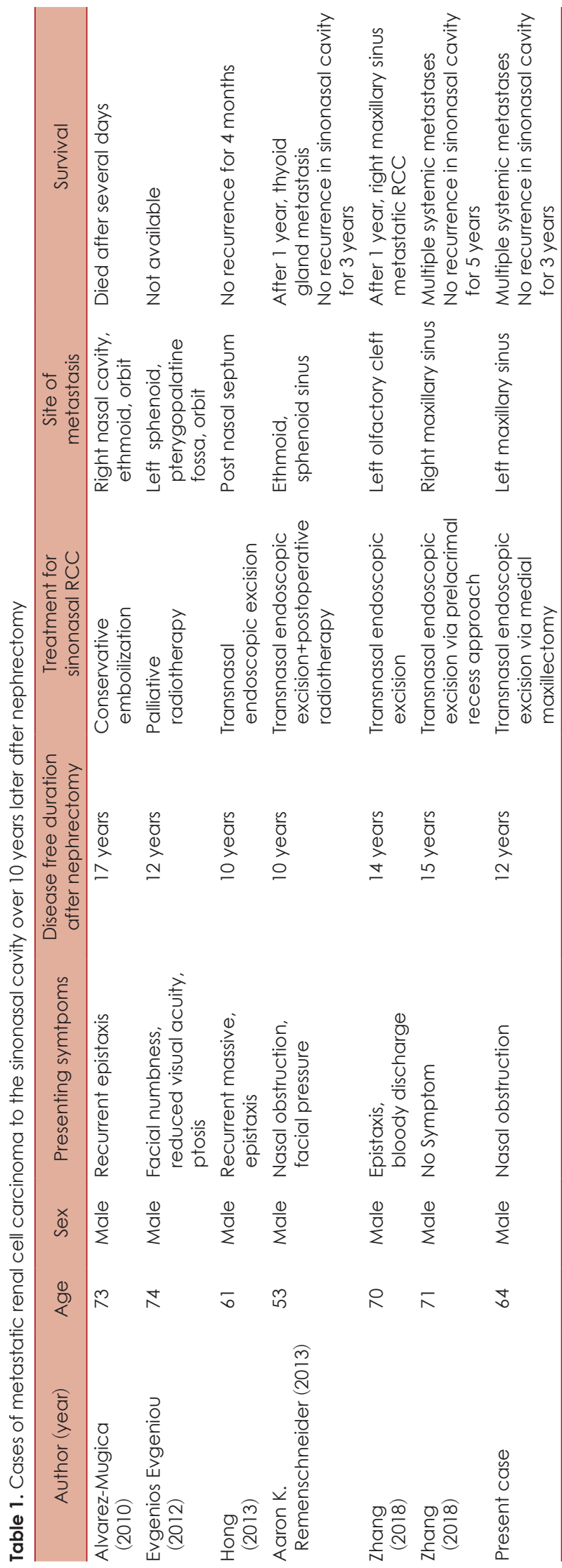

RCCs, which usually appear as multiple metastases, such as in the lung or liver, most metastatic tumors in nasal cavity or paranasal sinuses are single. ${ }^{19)}$ Therefore, endoscopic resection is recommended as the initial treatment in patients with a single resectable nasal or paranasal sinus RCC; this affords effective local control. In the reports on late recurrence listed in Table 1 and our present case, endonasal endoscopic resection induced a complete local response. Endoscopic resection is strongly recommended for patients without primary tumor recurrence if only a sinonasal RCC metastasis is evident. However, preoperative embolization should be considered because a metastatic RCC tumor can give rise to massive bleeding. Remenschneider et al. ${ }^{10)}$ reported that patients with metastatic sinonasal RCC successfully treated with preoperative embolization followed by endoscopic surgical resection with no significant blood loss. Furthermore, external approaches also should be considered in cases in which it is difficult to operate endoscopically.

Despite various treatment options, the prognosis for metastatic sinonasal RCC is poor. However, the 5-year survival rate for metastatic RCC remains as high as $20 \%$ compared to the mean survival time of about 5.7 months for overall metastatic sinonasal carcinoma originated from other organs such as lung, breast or prostate. ${ }^{320)}$ Radiotherapy may be considered if a metastatic tumor abuts the cranial nerve, if a high risk of massive intraoperative hemorrhage is apparent, or if a patient is to undergo palliative treatment only. The effectiveness of postoperative radiotherapy remains controversial; some studies reported that this provided no benefit. ${ }^{21)}$ Chemotherapy and targeted immunotherapy can be used to treat multiple metastases and unresectable tumors. In our case, we prescribed pazopanib and axitinib, a potent and selective multi-targeted receptor tyrosine kinase inhibitor multikinase inhibitor, to treat the multiple systemic metastases.

Sinonasal RCC is rare and late metastases are extremely rare, especially in patients lacking primary tumor recurrence. Early detection and diagnosis might improve survival. Careful endoscopic examination is recommended when a patient with an RCC history presents with a nasal symptom. A careful biopsy can be performed after CT or MRI examination.

\section{CONCLUSION}

When a patient with a history of RCC presents with uni- 
lateral epistaxis or a nasal obstruction, and a hypervascular sinonasal mass is evident on imaging, metastatic RCC should be considered, although the patient may have undergone complete primary tumor resection over a decade earlier. It is important to devise an appropriate treatment plan according to whether there are multiple or localized metastases, and whether the metastatic tumors are resectable.

\section{REFERENCES}

1) Motzer RJ, Bander NH, Nanus DM. Renal-cell carcinoma. N Engl J Med 1996;335(12):865-75.

2) Simo R, Sykes AJ, Hargreaves SP, Axon PR, Birzgalis AR, Slevin NJ, et al. Metastatic renal cell carcinoma to the nose and paranasal sinuses. Head Neck 2000;22(7):722-7.

3) Mekhail TM, Abou-Jawde RM, Boumerhi G, Malhi S, Wood L, Elson $\mathrm{P}$, et al. Validation and extension of the Memorial Sloan-Kettering prognostic factors model for survival in patients with previously untreated metastatic renal cell carcinoma. J Clin Oncol 2005;23(4): 832-41.

4) Ballarin R, Spaggiari M, Cautero N, De Ruvo N, Montalti R, Longo $\mathrm{C}$, et al. Pancreatic metastases from renal cell carcinoma: the state of the art. World J Gastroenterol 2011;17(43):4747-56.

5) Batsakis JG, McBurney TA. Metastatic neoplasms to the head and neck. Surg Gynecol Obstet 1971;133(4):673-7.

6) Sountoulides P, Metaxa L, Cindolo L. Atypical presentations and rare metastatic sites of renal cell carcinoma: a review of case reports. $\mathrm{J}$ Med Case Rep 2011;5:429.

7) Thomas JS, Kabbinavar F. Metastatic clear cell renal cell carcinoma: a review of current therapies and novel immunotherapies. Crit Rev Oncol Hematol 2015;96(3):527-33.

8) Ljungberg B, Campbell SC, Choi HY, Jacqmin D, Lee JE, Weikert S, et al. The epidemiology of renal cell carcinoma. Eur Urol 2011; 60(4):615-21.
9) Boles R, Cerny J. Head and neck metastases from renal carcinomas. Mich Med 1971;70(16):616-8.

10) Remenschneider AK, Sadow PM, Lin DT, Gray ST. Metastatic renal cell carcinoma to the sinonasal cavity: a case series. J Neurol Surg Rep 2013;74(2):67-72.

11) Alvarez-Mugica M, Bulnes Vazquez V, Jalon Monzon A, Gil A, Rodriguez Robles L, Miranda Aranzubia O. Late recurrence from a renal cell carcinoma: solitary right maxilar mass 17 years after surgery. Arch Esp Urol 2010;63(2):147-50.

12) Hong SL, Jung DW, Roh HJ, Cho KS. Metastatic renal cell carcinoma of the posterior nasal septum as the first presentation 10 years after nephrectomy. J Oral Maxillofac Surg 2013;71(10):1813 e1-7.

13) Zhang N, Zhou B, Huang Q, Chen X, Cui S, Huang Z, et al. Multiple metastases of clear-cell renal cell carcinoma to different region of the nasal cavity and paranasal sinus 3 times successively: a case report and literature review. Medicine (Baltimore) 2018;97(14):e0286.

14) Zisman A, Pantuck AJ, Wieder J, Chao DH, Dorey F, Said JW, et al. Risk group assessment and clinical outcome algorithm to predict the natural history of patients with surgically resected renal cell carcinoma. J Clin Oncol 2002;20(23):4559-66.

15) McNichols DW, Segura JW, DeWeerd JH. Renal cell carcinoma: long-term survival and late recurrence. J Urol 1981;126(1):17-23.

16) Friberg S, Nystrom A. Cancer Metastases: Early Dissemination and Late Recurrences. Cancer Growth Metastasis 2015;8:43-9.

17) Bastier PL, Dunion D, de Bonnecaze G, Serrano E, de Gabory L. Renal cell carcinoma metastatic to the sinonasal cavity: a review and report of 8 cases. Ear Nose Throat J 2018;97(9):E6-E12.

18) Razek AA, Huang BY. Soft tissue tumors of the head and neck: imaging-based review of the WHO classification. Radiographics 2011; 31(7):1923-54.

19) Ather MH, Masood N, Siddiqui T. Current management of advanced and metastatic renal cell carcinoma. Urol J 2010;7(1):1-9.

20) Abi-Fadel F, Smith PR, Ayaz A, Sundaram K. 2012. Paranasal sinus involvement in metastatic carcinoma. J Neurol Surg Rep 2012; 73(1):57-9.

21) Ziari M, Shen $S$, Amato RJ, Teh BS. Metastatic renal cell carcinoma to the nose and ethmoid sinus. Urology 2006;67(1):199. 